\title{
Photo Alignment Behaviour of Polyethene Imine Having Cyano Azobenzene Side Chain Mohammad Kamruzzaman ${ }^{1 *}$ and Seiji Kurihara ${ }^{2}$. \\ ${ }^{1}$ Department of Applied Chemistry and Chemical Engineering, Dhaka University, Dhaka-1000, Bangladesh. ${ }^{2}$ Department of Applied Chemistry and Biochemistry, Kumamoto University, Kurokami 2-39-1, Kumamoto 860-8555, Japan.
}

(Received: 12 March 2013; Accepted: 23 December 2014)

\begin{abstract}
New type of polyethene imine having cyano (-CN) side chain through six methylene spacer group (PEI6C) was successfully synthesised and characterised. The effect of reaction parameters on degree of substitution of PEI6C was also studied and the reaction conditions were optimised. Photochemical as well as photoorientational behaviour of the polymer were investigated. PEI6C in solid film exhibited photoresponsive properties upon irradiation with UV and visible light. PEI6C film also exhibited reversible molecular orientation from random state to out-of-plane and from out-of-plane to random state upon non-polarised visible and UV light irradiation.
\end{abstract}

Keywords: Azobenzene polymer, Polyethene imines, Photo alignment behaviour, Out-of-plane orientation.

\section{Introduction}

Azobenzene containing polymers have attracted considerable attention for the possibility of changing the molecular orientation by irradiation with an appropriate wavelength of light, and the potential applications in reversible optical storage, holographic grating and optical switching ${ }^{1,2}$. Azobenzene derivatives are known to undergo cis/trans photo-isomerisation upon irradiation. There are two typical liquid crystalline systems in relating to the photo-cotnrol of the molecular orientation by means of photoisomerisation of azobenzene compounds: one is "photochemical phase transition" between a liquid crystalline phase and a random state $^{3-5}$, the other is photoalignment between in-plane and out-of-plane structures, which is called command surface ${ }^{1,6}$. Very few papers have been published on spontaneous outof-plane molecular orientation of polymeric liquid crystals (PLCs) $^{7-11}$. Bobrovsky group ${ }^{7}$ reported that polyacrylate copolymer containing 4-ethoxy-4'-hexoxyazobenzene and cholesterol groups as side chains showed spontaneous outof-plane molecular orientation on a glass substrate by annealing at $75^{\circ} \mathrm{C}$, whereas only partial out-of-plane molecular orientation was observed for polyacrylate homopolymer. We also reported that PEI having nitro substituent azobenzene side chain group showed out-ofplane molecular orientation on a glass substrate by annealing but not upon non-polarized visible light irradiation $^{12}$. In addition, Ujiie group ${ }^{13,14}$ reported that some polyethene imines (PEIs) having azobenzene as side groups show liquid crystalline phases and align perpendicular to the substrate spontaneously by heating and followed by cooling without any alignment process. So, by photochemically controlling the molecular orientation of PEIs between random state and out-of-plane structures, one can fabricate the optical switching system showing excellent memory stability by coating PEIs on a substrate without any alignment process.

In this article, we synthesised polyethene imine having cyano azobenzene side chain group through six methylene spacer group (PEI6C) and investigated its photochemical as well as photo alignment behaviour on non-polarised visible and UV light irradiation.

\section{Materials and Methods}

\section{Materials}

4-Cyano phenylamine, 1,6-Dibromohexane and polyethene imine of low $(\mathrm{Mn} \approx 1800)$ and high $(\mathrm{Mn} \approx 10,000)$ molecular weight were used without further purification. $N, N$-dimethylformamide (DMF) was dried with molecular sieves prior to use.

\section{Synthesis of azobenzene monomer and polymer}

4-Cyano-4'-hydroxyazobenzene (CAz-OH): An aqueous solution $\left(30 \mathrm{~cm}^{3}\right)$ of $\mathrm{NaNO}_{2}(3.1 \mathrm{~g}, 45 \mathrm{mmol})$ was added dropwise to another solution containing 4-cyano phenylamine (5.3 g, $45 \mathrm{mmol})$ and concentrated $\mathrm{HCl}(14 \mathrm{~mL})$ at $5^{\circ} \mathrm{C}$. A solution of phenol $(4.7 \mathrm{~g}, 50 \mathrm{mmol})$ in aqueous $\mathrm{NaOH}(2 \mathrm{M}$, $75 \mathrm{~mL}$ ) was then added at the same temperature. The mixture was then stirred for a further period of $2 \mathrm{~h}$. The precipitate formed was filtered and washed to neutral with water. The separated precipitate was then suspended in ethanol ( $50 \%$ aq.) and acidified ( $\mathrm{HCl}$ ) when precipitate of $\mathrm{CAz}-\mathrm{OH}$ was formed. The precipitated orange colour product was filtered, recrystallised from ethanol twice and dried under vacuum.

Yield: $61.5 \%$ as solid. Melting point: $202-203^{\circ}$ C. ${ }^{1} \mathrm{H}-\mathrm{NMR}$ $\left(\mathrm{CDCl}_{3}, \delta\right): 7.83(2 \mathrm{H}, \mathrm{dd}$, aromatic), $8.21(2 \mathrm{H}, \mathrm{dd}$, aromatic), $7.92(2 \mathrm{H}, \mathrm{d}$, aromatic), $6.88(2 \mathrm{H}, \mathrm{d}$, aromatic), $5.28(1 \mathrm{H}, \mathrm{s},-$ $\mathrm{OH}$ ). Anal. Calcd for $\mathrm{C}_{13} \mathrm{H}_{9} \mathrm{~N}_{3} \mathrm{O}$ (223.23): C- 69.95; H- 4.06; N- 18.82. Found: C- 70.10; H- 4.22; N- 18.81.

4-(6-Bromo-n-hexyloxy)-4'-cyano Azobenzene [CAzO6Br]: 4(6-Bromo-n-hexyloxy)-4'-cyano azobenzene was synthesizsd by the following procedure: To a mixture of $0.98 \mathrm{~g}$ (4.4 $\mathrm{mmol})$ of 4- cyano-4'-hydroxyazobenzene (CAz-OH) and $0.91 \mathrm{~g}(6.6 \mathrm{mmol})$ potassium carbonate dissolved in $150 \mathrm{~mL}$ of dried acetone, $22.3 \mathrm{mmol}$ of 1,6-dibromohexane was added. After refluxing for $48 \mathrm{~h}$ at $70^{\circ} \mathrm{C}$ the reaction mixture was filtered and evaporated the solvent from filtrate and the orange colour product $\mathrm{CAzO} \mathrm{Br}$ was re-crystallised twice from methanol. The reaction scheme is shown in Scheme 1.

*Author for correspondence. email: kamruzzamanm@du.ac.bd 

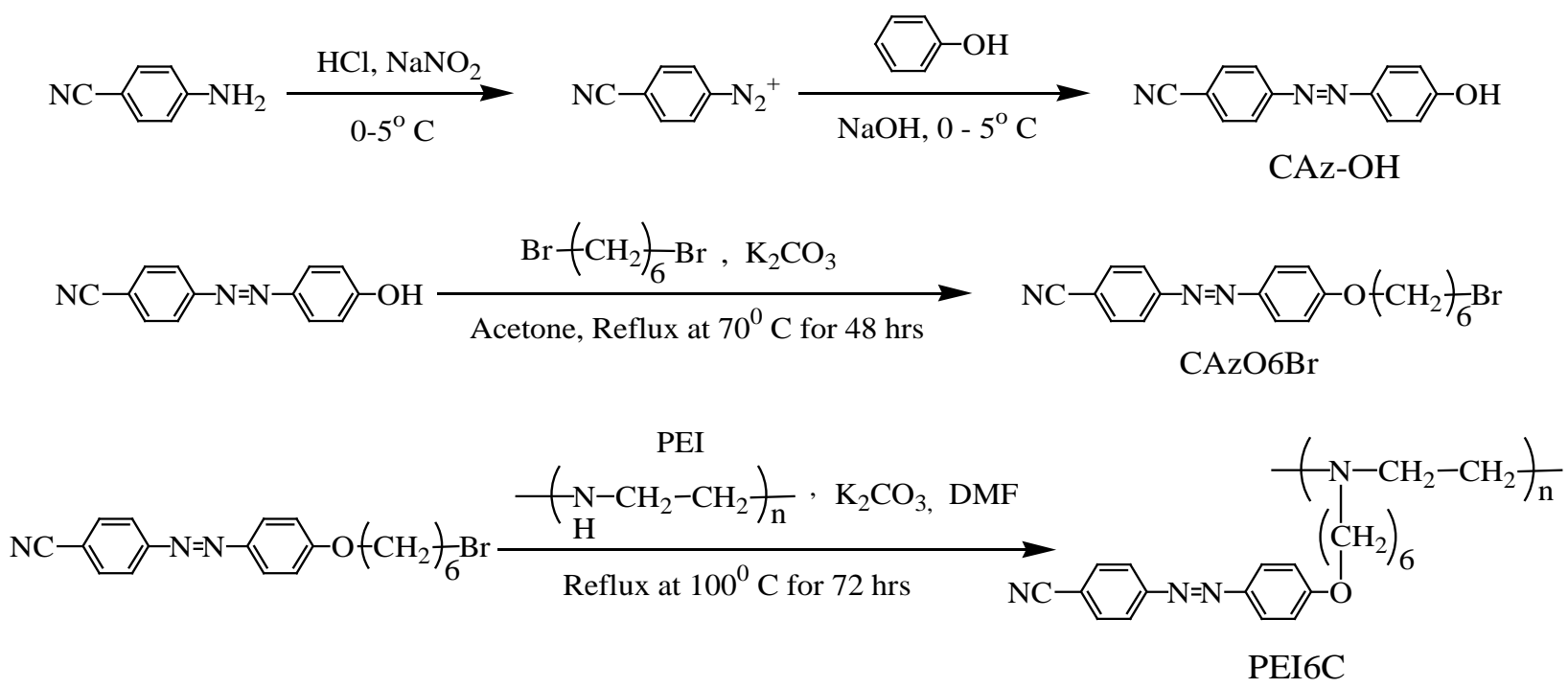

Scheme 1. Synthetic route for monomer and polymer.

Yield: $67.4 \%$ as solid. Melting point: $132-134^{\circ} \mathrm{C}$. Anal. calcd for $\mathrm{C}_{19} \mathrm{H}_{20} \mathrm{BrN}_{3} \mathrm{O}$ (386.29): C- 59.08; H- 5.22; N10.88. Found: C- 59.94; H- 4.83; N- 10.54.

Azobenzene containing polyethene imine (PEI6C): Polyethene imines having cyano substituent azobenzene side chain group with six methylene spacer groups was synthesised using different reaction conditions. The typical procedure is as follows: 4-(6-Bromo- $n$-hexyloxy)-4'-cyano azobenzene $1.62 \mathrm{~g}$ ( $4.2 \mathrm{mmol})$, polyethene imine $0.18 \mathrm{~g}$ (4.0 mmol), potassium carbonate $1.8 \mathrm{~g}(6.0 \mathrm{mmol})$ and $50 \mathrm{~mL}$ dry DMF were added into a round bottom flask equipped with a condenser. With continuous stirring the reaction mixture was refluxed at $100^{\circ} \mathrm{C}$ for $72 \mathrm{~h}$. After the reaction, the mixture was filtered and $\sim 50 \%$ of DMF was evaporated from the filtrate and then poured into methanol. The resulting polymer (PEI6C) was purified by precipitation from chloroform/THF into methanol two to three times. The removal of the monomer was monitored using thin-layer chromatography. Finally, the product was dried in vacuum for $24 \mathrm{~h}$.

\section{Characterisation}

To confirm the structure and composition of the synthesised monomers and polymer, elemental analyses were performed with a YANAKO CHN CORDER MT-6. The nmr spectra were recorded in $\mathrm{CDCl}_{3}$ or acetone using TMS as an internal standard on a JEOL JNM-EX400, 400MHz NMRspectrometer.

The phase transition behaviour of the polymer was studied by differential scanning calorimetry (DSC; Seiko SSC5020) with a heating rate of $10 \mathrm{Kmin}^{-1}$ and polarising optical microscopy (Olympus BHSP polarising microscope; Mettler FP-80 and FP 82 hot stage and controller).

X-ray diffraction was used to determine the LC phases and determine the lattice spacing of the smectic layers. Rigaku, RINT 2100/PC XRD machine (X-Ray, 40kV/200mA) equipped with a $\theta-\theta$ wide angle goniometer and scintillation detector was used for X-ray diffraction (XRD) measurement.

Photoirradiation was performed by using a 500W highpressure Hg lamp with adequate cut filter for UV and visible light at room temperature. The orientational order was studied using polarised UV-Vis spectroscopy (Perkin Elmer Lambda $650 \mathrm{UV} / \mathrm{Vis}$ Spectroscopy) and the angular dependence of the absorbance was measured. The values of order parameter determined by spectroscopic method were calculated by Eq. $(1)^{7}$

$$
S=\frac{A \|-A \perp}{A \|+2 A \perp}
$$

where $A_{\|}$is the absorbance at the preferred direction; $A_{\perp}$ is the absorbance perpendicular to this direction.

\section{Results and Discussion}

\section{Synthesis and characterisation of PEI6C}

Liquid crystalline polyethene imine having methoxy substituent azobenzene side chain (PEI6C) was successfully synthesised. For a good photo alignment behaviour, polyethene imines should have high degree of substitution. It is assumed that the introduction of azobenzene group into polyethene imine main chain increase the out-of-plane alignment behaviour of the polymers. So in synthetic step, to achieve higher degree of substitution in PEI, reaction conditions were optimised by changing the reaction parameters such as temperature, reaction time, molar ratio of azobenzene monomer and PEI, molecular weight of PEI and reaction media. The degree of substitution of PEI6C has considerable effect on all of these parameters. With the increase in reaction temperature and reaction time, the degree of substitution increased, and best results were obtained at a temperature of $100^{\circ} \mathrm{C}$ and reaction time of 72 
h. At temperature more than $100^{\circ} \mathrm{C}$ and longer reaction time (above $72 \mathrm{~h}$ ), the degree of substitution increased slightly but the solubility of PEI6C decreased probably due to the formation of a gel. Finally we synthesised PEI6C having degree of substitution 63.7 by optimising the reaction conditions such as temperature $100^{\circ} \mathrm{C}$, reaction time $72 \mathrm{~h}$, molar ratio of azobenzene monomer to PEI $(\mathrm{Mn} 1800)=4.2: 4.0$, and solvent $(\mathrm{DMF})=50 \mathrm{~cm}^{3}$. Synthesised polymer showed good solubility in DMF, $\mathrm{CHCl}_{3}$, THF and cyclohexanone.

Thermal characteristics of synthesised PEI6C was studied using differential scanning calorimetry (DSC) with a heating rate of $10 \mathrm{Kmin}^{-1}$ and polarised optical microscopy (POM) analyses. On DSC thermogram, PEI6C showed two endothermic peaks with low glass transition temperature (G 38 S $115 \mathrm{~N} 147$ I). On the basis of the DSC and POM measurements, these two peaks were assigned to smecticnematic, and nematic-isotropic thermal phase transitions. The polymer is stable up to $215^{\circ} \mathrm{C}$.

The synthesised polymer was also characterised by X-ray diffraction measurement. The X-ray diffraction patterns for synthesised polyethene imine film confirmed that the polymer is smectic layer structures and exhibited few strong diffraction peaks in small angle region. On the basis of $\mathrm{X}$ ray diffraction patterns, the smectic layer spacings were calculated from the XRD peaks using Bragg's law and the

(a)

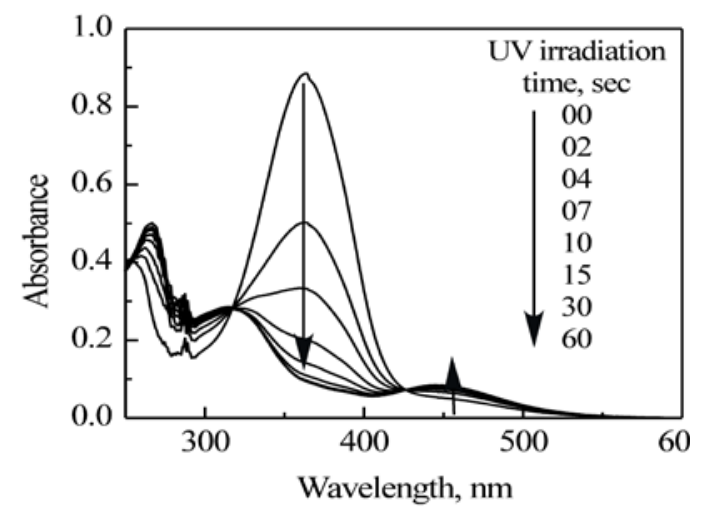

Photoinduced alignment behavior of PEI6C in solid film

The photo alignment behaviour and photochemical behaviours of PEI6C in solution and in solid state were examined. The changes in the UV-vis. absorption spectra of PEI6C in THF are shown in Figure 1. Before irradiation, PEI6C in THF showed a strong absorption at $352 \mathrm{~nm}$ and a weak absorption around 460 $\mathrm{nm}$, corresponding to $\pi \rightarrow \pi^{*}$ transition and $\mathrm{n} \rightarrow \pi^{*}$ transition of the azo-chromophores, respectively. UV irradiation caused decrease and increase in absorption at 352 and $460 \mathrm{~nm}$, respectively, indicating photoisomerisation from trans $\rightarrow$ cis form. It became photostationary in $10 \mathrm{sec}$ in case of trans-cis photoisomerisation by UV light irradiation and $30 \mathrm{sec}$ in case of cis-trans isomerisation by visible light irradiation. Photoisomerisation of the polymer in solution shows isosbestic points, indicating uniform photoreactions. The subsequent cistrans isomerisation by visible light irradiation rebuilt the initial state completely, which demonstrates the reversibility of the reaction. Therefore, the irradiation of light in this wavelength region should cause the photoisomerisation from the transform to the cis-form and vice-verse for the cis-form. Consequently, trans-cis-trans photoisomerisation cycle of PEI6C will be brought about by irradiation of the light in this region $^{15-17}$.

(b)

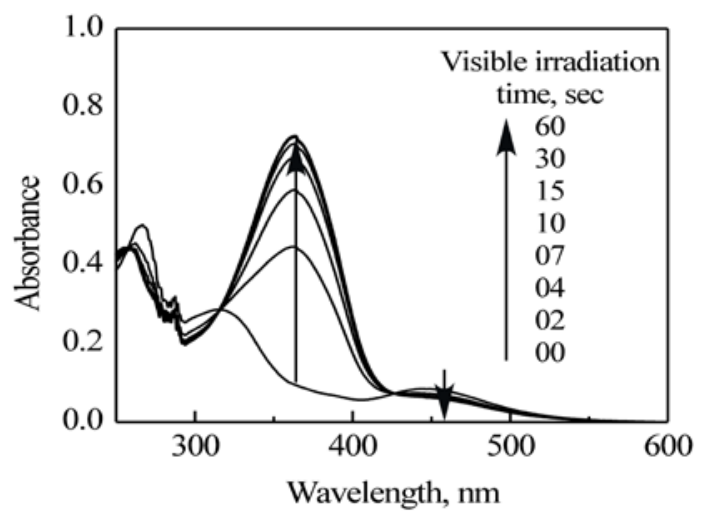

Fig. 1. Changes in UV-Vis. absorption spectra of PEI6C in THF by irradiation of (a) UV (365 nm) and (b) Visible light $(435 \mathrm{~nm})$. The intensity of UV and visible light was $7.0 \mathrm{mWcm}^{-2}$ and $80 \mathrm{mWcm}^{-2}$ respectively.

results are $2 \theta(1)=2.28 ; 2 \theta(2)=5.68$; $\operatorname{dist} .1(\AA)=31.30$ and $\operatorname{dist.2}(\AA)=15.54$.

In order to understand photochemical and photoinduced optical anisotropy in polymeric films, the spectral changes were investigated in polymeric film under irradiation with UV and non-polarised visible light of different wavelengths. Depending on the irradiation wavelength, different steady states of cis-trans photo-isomerisation were established allowing or preventing the photo-orientation of azobenzene chromophores in PEI6C.

In this study, all experiments were performed for polymeric films with a thickness ranging from 200 to $300 \mathrm{~nm}$. The films were prepared by spin coating technique. Upon irradiation of non polarised UV light on spin coated solid films of PEIs, significant spectral changes were observed in 
polymers which are typical for trans-cis photoisomerization $^{17-19}$. In PEI6C film, photostationary state with a little high content of the cis isomeric form was achieved within 2-3 min as shown in Figure 2(a). Due to molecular aggregation, the rate of trans-cis photoisomerisation in film is not as high as that in solution. On the subsequent irradiation with visible light of $435 \mathrm{~nm}$, the reverse changes occurred establishing a steady state with a high concentration of trans isomers [Figures 2(b)]. The recovery of the initial spectrum was complete, even the value of absorption maxima corresponding to $\pi \rightarrow \pi^{*}$ transition exceeded the initial value. This phenomenon was related to the partial out-of-plane molecular ordering of azobenzene moieties on glass substrate.

Therefore, to quantify the photo induced anisotropy in PEI6C solid film, polarised absorption spectra were recorded and explored the angular dependency of
Upon prolonged irradiation (>10s), one may observe a decrease in the absorption in both regions corresponding to the $\pi \rightarrow \pi^{*}$ and $n \rightarrow \pi^{*}$ transitions as shown in Figures 2(c). Within the first $10 \mathrm{sec}$ of irradiation with visible light, the back cis-trans photoisomerisation occurred, while upon continued irradiation, the azobenzene groups were oriented along the normal of film due to photo-orientation ${ }^{7}$. In this case, the transition moment of these groups become oriented perpendicular to the plane of films; as a result, the probability of light absorption and, thus, optical density markedly decreased ${ }^{7}$. So irradiation with non-polarised visible light $(435 \mathrm{~nm})$ caused the induction of anisotropy in the films by photoorientation of azobenzene side groups. (a)

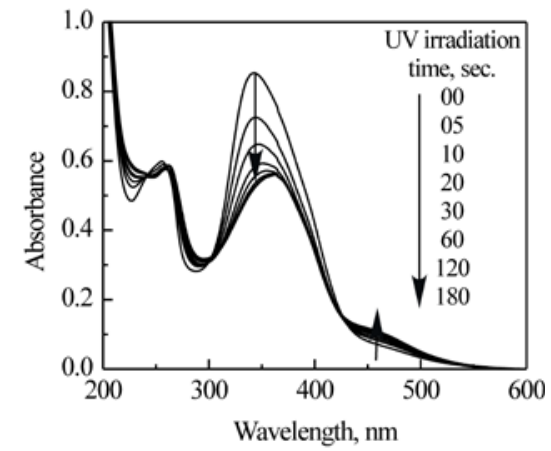

(b)

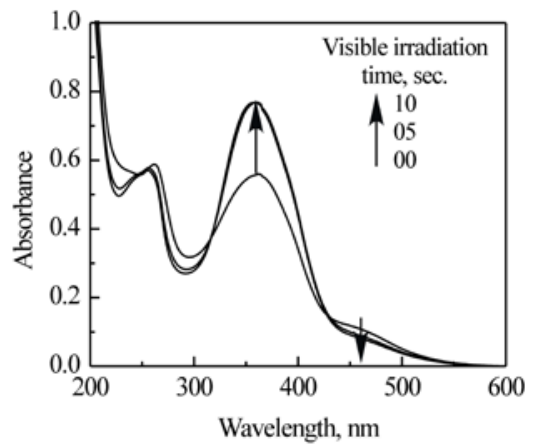

(c)

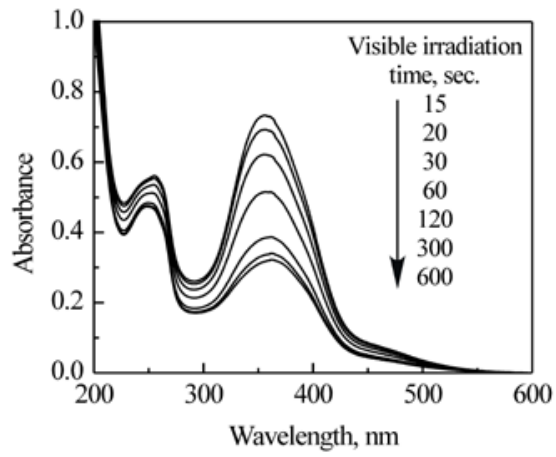

Fig. 2. Changes of absorption spectra of PEI6C polymeric film during (a) UV irradiation (365 nm), (b) first 10 sec visible light irradiation and (c) further visible light irradiation (435 nm). The intensity of UV and visible light was $7.0 \mathrm{mWcm}^{-2}$ and $80 \mathrm{mWcm}^{-2}$ respectively.

absorbance before and after irradiation. Irradiation of visible light on PEI6C film brought about not only by decrease in absorbance corresponding to the $\pi \rightarrow \pi^{*}$ transition without polariser, but also by change in the polarised absorption spectra as shown in Figure 3. The order parameter, the degree of out-of-plane ordering, was calculated from polarised absorption spectra by using equation (1).

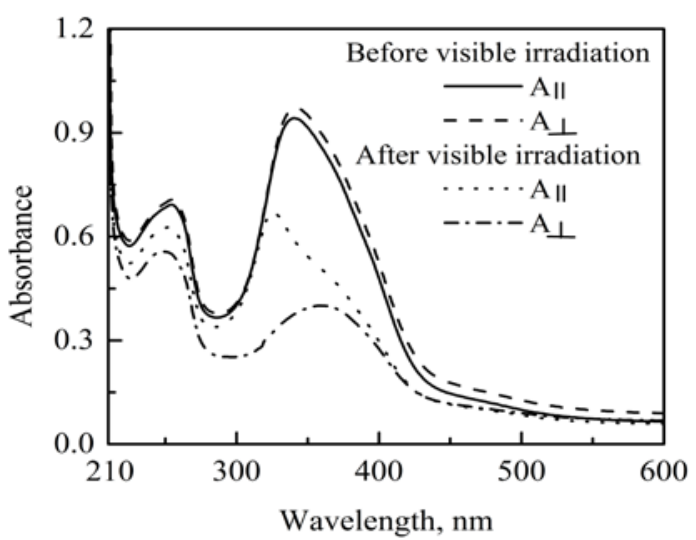

Fig. 3. Polarized UV-vis. absorption spectra of PEI6C single layer film before and after irradiation of non-polarized visible light (435 $\mathrm{nm}$ ) during $5 \mathrm{~min} . A_{/ /}$and $A_{\perp}$ denote absorption parallel and perpendicular to the polarization direction of actinic light respectively. Spectral measurements were made at $45^{\circ}$ to film normal.
The polarised absorption spectra were recorded at an angle of $45^{\circ}$ to the normal of the film, and by following rotation of the film for polar plot to evaluate the quantity of out-ofplane molecular orientation. From polarised absorption spectra, polar plot for polymeric film was also plotted and is shown in Figure 4. After irradiation of non-polarised visible light, the absorbance parallel to the polarisation direction of actinic light was higher than that perpendicular to the polarisation direction of actinic light. From polar plot, it is clear that, before irradiation, no angular dependency was observed, indicating that the azobenzene chromophores were randomly orientated on glass substrate in solid film. But after irradiation, PEI6C solid film showed big angular dependency and the shape of the corresponding polar plot is concave, allowing to assess the level of order parameter ${ }^{7}$. The out-of-plane order parameter values before and after irradiation were 0.006 and 0.243 respectively. 
It was established that the photoorientation took place in the steady state of the photoisomerisation via numerous angularselective photo-isomerisation cycles leading to a preferred orientation of the azobenzene groups perpendicular to the electric field vector of the excitation light.

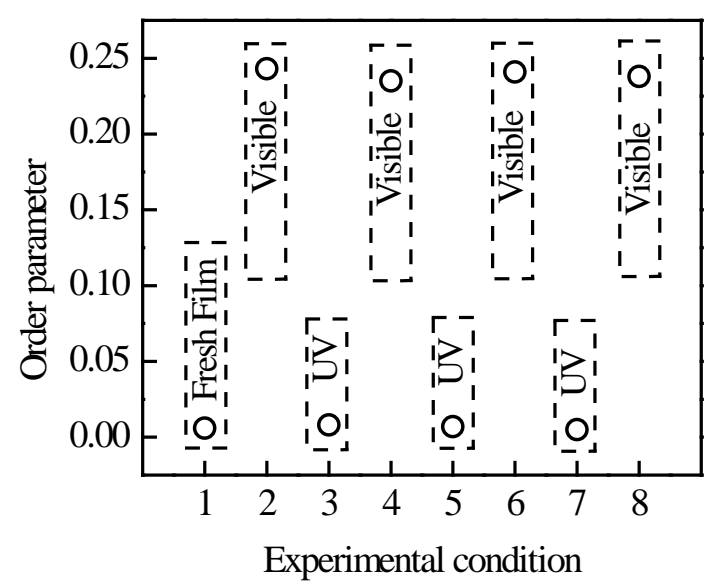

Fig. 5. Reversibility of alignment behavior of PEI6C at different experimental conditions.

Reversibility of alignment behavior of PEI6C in solid film

In order to show the possibility of reversible reorientation of azobenzene chromophores in PEI6C, molecular ordering behaviours were observed at different conditions. And

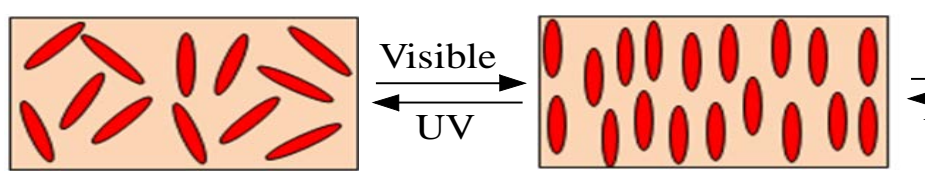

Scheme 2. Schematic representation of the alignment behavior of azobenzene different conditions.

polymeric film showed high order parameter value showing out-of-plane molecular alignment. Irradiation with the 365 $\mathrm{nm}$ light brings the film always into the isotropic state and erases any previously induced anisotropy. So after the irradiation of UV light on non-polarised visible light irradiated films, the value of order parameter decreased via cis-trans photoisomerisation and reached to almost initial level. Namely the azobenzene molecules aligned randomly on the glass substrate. Following non-polarised visible light irradiation again increased the order parameter value, indicating the transformation of molecular orientation of azobenzene molecules from random state to out-of-plane order due to photo-orientation. Again upon UV irradiation, the azobenzene group aligned randomly on the glass substrate (Scheme 2). It should be noted that after each step of irradiation with the $365 \mathrm{~nm}$ UV light the angulardependent spectra were exactly the same. This indicates a complete erasure of the previously written orientation was achieved. 


\section{Conclusion}

Liquid crystalline polyethene imine (PEI6C) having cyano (-CN) azobenzene side chain through methylene spacer group was successfully synthesised and characterised by DSC, Polarised Optical Microscopic and XRD analysis. The effect of reaction parameters on degree of substitution of PEI6C were also studied and optimised the reaction conditions. PEI6C, both in solution and in solid film exhibited photoresponsive properties upon irradiation of UV and visible light. PEI6C film also exhibited reversible molecular orientation from random state to out-of-plane and from out-of-plane to random state on non-polarised visible and UV light irradiation. The reversible molecular ordering of azobenzene chromophores showed that optical anisotropy in the film can be completely erased and rewritten without any memory effect of the previous irradiation.

\section{References}

1. Ichimura, K, 2000. Photoalignment of liquid-crystal systems. Chem Rev. 100, 1847-1873.

2. Ikeda, T., 2003. Photomodulation of liquid crystal orientations for photonic applications, J. Mater. Chem. 13, 2037-2057.

3. Ikeda, T. D. B. Horiuchi, D. B. Karanjit, S. Kurihara and S. Tazuke, 1990. Photochemically induced isothermal phase transition in polymer liquid crystals with mesogenic phenyl benzoate side chains. 1. Calorimetric studies and order parameters. Macromolecules. 23, 36-42.

4. Ikeda, T and O. Tsutsumi, 1995. Optical switching and image storage by means of azobenzene liquid-crystal films. Science. 268, 1873-1875.

5. Kurihara, S. D. Yoneyama and T. Nonaka, 2001. Photochemical switching behavior of liquid-crystalline networks: Effect of molecular structure of azobenzene molecules. Chem Mater. 13, 2807-2812.

6. Ichimura, K., Y. Suzuki and T. Seki, 1988. Reversible change in alignment mode of nematic liquid crystals regulated photochemically by command surfaces modified with an azobenzene monolayer. Langmuir. 4, 1214-1216.

7. Bobrovsky, A., N. Boiko, V. Shibaev and J. Stumpe, 2004. Comparative study of photoorientation phenomena in photosensitive azobenzene-containing homopolymers and copolymers. J. Photochem. and Photobio A: Chem. 163, 347-358.

8. Sapich, B., A. B. E. Vix, J. P. Rabe, J. Stumpe, G. Wilbert and R. Zentel, 2006. Ordering and dewetting in spin-coated films of a liquid crystalline main chain polymer. Thin Solid Films. 514, 165-173.
9. Uekusa, T., S. Nagano and T. Seki, 2009. Highly ordered inplane photoalignment attained by the brush architecture of liquid crystalline azobenzene polymer. Macromolecules. 42, 312-318.

10. Ujiie, S. and K. limura, 1992. Thermal properties and orientational behaviour of a liquid-crystalline ion complex polymer. Macromolecules. 25, 3174-3178.

11. Ujiie, S. and K. Iimura, 1993. Formation of smectic orientational order in an ionic thermotropic liquid-crystalline side-chain polymer. Poly. J. 25, 347-354.

12. Kamruzzaman, M., Y. Kuwahara, T. Ogata, S. Ujiie and S. Kurihara, 2011. Synthesis, thermal, and photo alignment behaviour of polyethene imines having nitro substituent azobenzene side chain group. J. Appl. Polym. Sci. 120, 950-959. 
13. Ujiie, S. and K. Iimura, 1990. Ammonium halide type thermotropic liquid-crystalline polyetheneimines and those low-mass model compounds. Chem. Lett. 19, 995-998.

14. Ujiie, S. and Y. Yano, 2000. Thermotropic and lyotropic behaviour of novel amphiphilic liquid crystals having hydrophilic poly(ethyleneimine) units. Chem. Commun. 1, 79-80.

15. Fabian, J. and H. Hartmann, 1980. Light Absorption of Organic Colarants; Springer-Verlag: Berlin, pp 32-79.

16. Freiberg, S., F. L. Labarthet, P. Rochon and A. Natansohn, 2003. Synthesis and characterization of a series of azobenzene-containing side-chain liquid crystalline polymers. Macromolecules. 36, 2680-2688.

17. Alam, M. Z., T. Ohmachi, T. Ogata, T. Nonaka and S. Kurihara, 2006. Photoisomerisation behaviour and photoinduced surface relief gratings on azopolymer film by a monochromatic light irradiation. Optical Materials. 29, 365-370.

18. Natansohn, A. and P. Rochon, 2002. Photoinduced motions in azo-containing polymers. Chem. Rev. 102, 4139-4175.

19. Ishiguro, M., D. Sato, A. Shishido, and T. Ikeda, 2007. Bragg-type polarisation gratings formed in thick polymer films containing azobenzene and tolane moieties. Langmuir. 23, 332-338. 